\section{What is already known on this topic}

The new general medical services contract takes no account of deprivation or ethnicity on target levels

Women are least likely to receive adequate care for coronary heart disease

\section{What this study adds}

Practices in areas of high deprivation and high ethnicity will find it harder to meet targets in the new general medical services contract

Women are less likely than men to receive adequate care for diabetes

services contract. Our findings confirm the inequalities between the sexes reported by patients with diabetes and observed for patients with coronary heart disease. ${ }^{2}$

The association of deprivation and ethnicity with achievement of targets was substantial and was not explained by age, sex, or practice. Of the 17 quality indicators, 10 were adversely associated with deprivation and nine were adversely associated with ethnicity.

We found a large variation between practices in the recording of most of the indicators. Our study design prevented us from determining whether this was due to variation in the quality of care or to differences in the completeness of data entry, although electronic records tend to be more complete than paper records. ${ }^{3}$ The prevalence of diabetes in our study was higher than that in other studies in primary care. ${ }^{4}$ This might be because the data are recent and the prevalence of diabetes is increasing. Levels of recording of laboratory investigations were higher than clinical measures such as neuropathy testing. This might be because laboratory test results are now sent electronically to most practices and are automatically uploaded into the patients' clinical records, whereas clinical measurements are entered manually.
These data, reported at the start of the new general medical services contract, will be of interest both to practices as they plan their delivery strategies and to health service planners responsible for monitoring and remuneration. The large variation between practices in levels of outcomes achieved was expected, although the overall values were lower than expected, indicating the huge amount of work needed to provide optimum care for all patients. Practices in areas of high deprivation and high ethnicity will have to work harder to achieve the quality indicators for diabetes, and it is possible that those practices which most need the resources are the ones least likely to get them.

We thank David Stables (medical director of EMIS); Mike Pringle for help in creating QRESEARCH; the National Advisory Board for setting and monitoring policy; and the practices for contributing data. The QRESEARCH database is available at www.qresearch.org

Contributors: JH-C initiated and designed the study, obtained ethical approval, and undertook the data extraction, manipulation, and analysis; she is guarantor. SO'H contributed to the design, advised on the general medical services contract queries, and contributed to the paper. CC contributed to the design, advised and checked the statistical analysis, and contributed to the interpretation and the paper.

Funding: Grant from Trent NHS Executive.

Competing interests: QRESEARCH is a non-profit making organisation established to give good access to high quality data for research. JHC is one of the custodians of QRESEARCH; publication of this paper is likely to lead to increased awareness and usage of the database. Practices contributing data are not paid but receive feedback on quality measures. SO'H is a clinical design director for EMIS

Ethical approval: Trent multicentre ethics committee

1 Department of Health. National service framework for diabetes-the delivery strategy. London: DoH, 2003.

2 Hippisley-Cox J, Pringle M, Crown N, Meal A, Wynn A. Sex inequalities in ischaemic heart disease in general practice: cross sectional survey. BMJ 2001;322:832.

3 Hippisley-Cox J, Pringle M, Cater R, Wynn A, Hammersley V, Coupland $\mathrm{C}$, et al. Electronic record in primary care-regression or progression? Cross sectional survey. BMJ 2003;326:1439-43.

4 Newnham A, Ryan R, Khunti K, Majeed A. Prevalence of diagnosed diabetes mellitus in general practice in England and Wales. Health Stat $Q$ 2002;14:5-13.

(Accepted 29 June 2004)

doi $10.1136 /$ bmj.38279.588125.7C

\title{
Commentary: Socioeconomic inequalities in indicator scores for diabetes: poor quality or poor measures?
}

P G Shekelle

Hippisley-Cox et al report on scores for quality indicators for diabetes among a large number of UK patients and practices and how these vary depending on sex, ethnicity, and material deprivation. ${ }^{1}$ For many of the indicators, scores were worse for women and worse in practices with a high proportion of ethnic patients and those with high levels of material deprivation. This revelation of worse measures of care for women, poor people, and people from ethnic minority groups is not unique; there is a robust literature in America showing these same findings across numerous conditions, ${ }^{23}$ and similar findings have been reported in England. ${ }^{4}$ The question relevant to policy, particularly in light of the new general practitioner contract, is what do these differences in scores mean?

In America, these differences are usually interpreted as meaning that lower scores denote poorer quality of care. Should the same be true in the United Kingdom? For the most part, I think the answer is yes. The exception is the four measures reported on by Hippisley-Cox et al that are outcomes (proportion of patients with $\mathrm{HBA}_{1 \mathrm{C}}$ values under $7.5 \%$ or under $10 \%$; blood pressure less than 145/85 mm Hg; serum cholesterol concentration less than $5 \mathrm{mmol} / \mathrm{l})$. Although these are undoubtedly good outcomes to strive for (since they are strongly related to health outcomes such as fewer
West Los Angeles Veterans Affairs Medical Center, 11301 Wilshire Blvd., Los Angeles, CA 90073, USA P G Shekelle professor shekelle@rand.org 
microvascular and macrovascular complications from diabetes), they are determined by many factors other than medical care. The problem of adjusting for case mix is one reason I do not generally favour the use of outcomes as comparative measures of quality.

Most of the measures reported by the authors, however, are process measures, are almost or entirely under the control of the doctor or practice, and are much less sensitive to the need to adjust for differences in case mix. For example, only a scale and a tape measure are needed to record body mass index, but it was done significantly less often in women, poor people, and people from ethnic minority groups. This was also the case for flu vaccination. It is hard to explain these findings other than by poorer quality of care.

So, what is to be done? I think that these data mostly show that women, poor people, and people from ethnic minority groups get poorer quality of care than do white men living in leafy areas. Some evidence shows that in the United Kingdom, financial incentives, such as those in the new general practitioner contract, will help reduce (although not eliminate) these disparities. ${ }^{5}$ I do, however, favour attempts to tweak the existing general practitioner contract to recognise that achieving the outcome targets will vary depending on where the practice is located. This could be done by weighting the payments for outcome indicators by some measure of deprivation. But this attempted tweak will come at a price: it will be more complex, for sure; it will precipitate arguments over who is deprived and how much extra the payment should be; and it may have unexpected consequences. Still, I believe the search for policy that best promotes quality, efficiency, and equity in health care is a dynamic one. The key here is both to perform adequate evaluations of the new general practitioner contract and to have policy makers who are willing to make changes when evidence accumulates that the policy is either not achieving its intended effect or producing unintended, and unwanted, side effects.

Competing interests: None declared.
Hippisley-Cox J, O'Hanlon S, Coupland C. Association of deprivation, ethnicity, and sex with quality indicators for diabetes: population based survey in 53000 patients in primary care in 2004 BMJ 2004:399:1267-9.

2 Fiscella K, Franks P, Gold MR, Clancy CM. Inequality in quality: addressing socioeconomic, racial, and ethnic disparities in health care. JAMA 2000;283:2579-84

3 Epstein AM, Ayanian JZ. Racial disparities in medical care. $N$ Engl J Med 2001;344:1471-3.

4 Hippisley-Cox J, Pringle M, Crown N, Meal A, Wynn A. Sex inequalities in ischeamic heart disease in general practice: cross sectional survey. $B M J$ 2001;322:832

5 Baker D, Middleton M. Does primary care reduce health inequalities? National primary care research and development centre executive summary No 29. npcrdc.man.ac.uk/publications/29.pdf (accessed 23 Aug 2004).

\section{Corrections and clarifications}

Hepatitis B infections

Through editorial and production errors, we completely mangled a measurement in this editorial by Nicholas J Beeching (6 November, 1059-60). In the second sentence of the third paragraph from the end (about anti-HBs antibody seroconversion) the correct measurement in parentheses should have read, "anti-HBs antibody concentration > $10 \mathrm{IU} / 1\left[\right.$ not $\left.1 \times 10^{4} \mathrm{IU} / \mathrm{l}\right]$."

ABC of sexual health: Gender related disorders Readers may have had difficulty contacting a support group mentioned in this $\mathrm{ABC}$ article by Kevan Wylie (11 September, pp 615-7). This is because in the "web extra" information (on bmj.com) about support networks for patients undergoing gender transition we failed to double the "e" in the URL for the Mermaids support group; it should be www.mermaids.freeuk.com.

Minerva

During editing we inadvertently switched the affiliations of the second and third authors of the picture item about milker's nodule in the Minerva of 13 November ( $p$ 1194). We correctly stated that both K F To and Antonio Chuh work at the Chinese University of Hong Kong, Prince of Wales Hospital, Hong Kong, China, but K F To is professor in the department of anatomical and cellular pathology and Antonio Chuh is part time assistant professor in the department of community and family medicine (not the other way round).

\section{bmjlearning.com}

\section{Multimedia learning}

"Early multimedia learning looked like a car that had been cobbled together from different scrap yards with components of different sizes, colours, models, and ages. It was a mongrel beast."'

So said Donald Clark, a leading authority in multimedia learning in Britain. He was referring to the early flood of learning websites that had loud music and equally loud colour. Videos were mixed with animation, and graphics popped up all over the screen. The designers loved it, but users were at first bemused and then distracted, and in the end they learnt little. So far at BMJ Learning, we have erred on the side of multimedia learning for a purpose-you don't need broadband to use the site. Text is still very useful-experienced readers can read at a rate of 300 words a minute, but everyone can read at their own pace. You can also search text quickly, and we can easily update it.

However, we are building more multimedia content on the site. This month we have published a module on psoriasis. About 2\% of UK adults have psoriasis, and our module outlines how to diagnose and manage patients in primary care. Traditionally, colour slides are used to support text, but here the pictures are at the core of the module. We show you pictures of patients with different types of psoriasis and then test and train your skills in diagnosing and managing the condition. Further pictures enable you to test your knowledge of some complications of psoriasis such as psoriatic arthropathy and erythrodermic psoriasis. The module also gives an update on recent advances in management such as the role of vitamin $\mathrm{D}$ analogues and tumour necrosis factor blockers.

To find out more, look at our new module on bmjlearning.com.

Kieran Walsh editorial registrar, BMJ Learning (bmjlearning@bmjgroup.com)

1 Clark D. White paper: Media rich is not always mind rich. Epic Group. www.epic.co.uk/content/resources/white_papers/media mix.htm (accessed 29 Sep 2004). 